\title{
The bloom of natural product chemistry in China
}

\author{
Qi-Yu Zheng ${ }^{1 *} \&$ Ang Li ${ }^{2 *}$ \\ ${ }^{1}$ National Natural Science Foundation of China, Beijing 100085, China \\ ${ }^{2}$ State Key Laboratory of Bioorganic and Natural Products Chemistry, Collaborative Innovation Center of Chemistry for Life Sciences, \\ Shanghai Institute of Organic Chemistry, Chinese Academy of Sciences, Shanghai 200032, China
}

Received July 3, 2016; accepted July 11, 2016; published online August 8, 2016

Citation: $\quad$ Zheng QY, Li A. The bloom of natural product chemistry in China. Sci China Chem, 2016, 59: 1059-1060, doi: 10.1007/s11426-016-0280-7

Natural product chemistry is an important area across chemistry, biology, and pharmaceutical sciences. The discovery of natural products through isolation and structural elucidation expands the chemical space of biologically active molecules in an unparallel fashion; numerous medicinally relevant compounds arise from natural product scaffolds. The chemical synthesis of natural products advances the development of organic chemistry from the theoretical, strategic, and methodological perspectives, and provides versatile access to natural product derivatives and mimics with improved pharmacological properties. The studies of natural product biosynthesis reveal how nature generates these fascinating structures in an incredibly efficient and selective manner, which promotes the development of genomics-based discovery and cell factory-based synthesis of natural products. The target identification and mechanistic studies of natural products elucidate the relationship between their structures and functions in biological systems, which facilitates the discovery of new therapeutic targets and rational design of lead compounds for drug development. Nowadays natural product chemistry has become a fruitful field with flavors of multi-disciplines, which extends far beyond the boundary of "chemistry" by a conventional definition.

China has a long history of clinical utilizations of traditional herbal medicines, which prove to be a rich source of biologically active natural products. Thus, natural product chemistry attracts remarkable attention of China's scientific

*Corresponding authors (email: zhengqy@nsfc.gov.cn; ali@sioc.ac.cn) community in modern times. A representative accomplishment is the discovery, structural elucidation, chemical synthesis, derivatization, and clinical therapy of antimalarial natural product artemisinin. If a wide definition of natural products that is not limited to secondary metabolites is allowed, the chemical syntheses of bovine insulin and yeast alanine transfer RNA can be counted as landmark events in this field as well. In the past three decades, China's progress on natural product chemistry has drastically accelerated. A number of excellent reviews [1-3] describe some of the advancements from the 1980 to 2010. In the past decade, China's natural product chemistry has entered its golden era. On the basis of a long-term incubation, the joint endeavors of chemists of many generations result in such a beautiful bloom.

In the spring of 2015, the National Natural Science Foundation of China organized a symposium on natural product chemistry. Nearly 20 scientists updated their research and discussed future directions of the sub-disciplines in this field, including natural product discovery, chemical synthesis, biosynthesis, and chemical biology. Based on the fruitful outcome of the symposium, we edit this special thematic issue for Science China Chemistry, hopefully to bring out the great potential that still lies ahead in natural product chemistry.

The special issue is composed of 15 articles, which can be classified into 5 categories: natural product isolation and structural elucidation, total synthesis, synthetic method development, biosynthesis, and target identification. In the first part, Yue et al. reviewed the natural products 
possessing strained small ring systems; $\mathrm{Pu}$ et al. disclosed the discovery of ent-kaurane and ent-abietane diterpenoids from Isodon phyllostachys; Xu et al. reported polyprenylated acylphloroglucinols from Hypericum henryi. In the second part, Yao and Zhong summarized the Michael addition-based cyclization strategy in Lycopodium alkaloid synthesis; Liang and Qiao reviewed the recent advances in terpenoid syntheses from China; $\mathrm{Li}$ and Lee highlighted the chemical synthesis of glycoproteins; Liu and Zhang reported the total synthesis of proposed structures of jiangrines $\mathrm{C}$ and D; Fan and coworkers disclosed a strategic study towards constructing the nine-membered azonane ring system of palhinine A. In the third part, Zhang and Chen reviewed the asymmetric construction of all-carbon quaternary centers in natural product synthesis from China; Jia and Tao summarized recent applications of $\mathrm{C}-\mathrm{H}$ bond activation in natural product synthesis; Gao and Qiu reviewed recent advances of radical and photo reactions in natural product synthesis; Hong and coworkers reported stereoselective construction of skipped polyol enabled by oxonia-Cope rearrangement and iodolactonization. In the fourth part, Liu, Chen, and Lin reviewed biosynthesis-based artificial evolution of microbial natural products; Lin et al. disclosed that indole methylation protects diketopiperazine configuration in the maremycin biosynthetic pathway. In the last part, Lei et al. summarized recent advances in target identification by natural product based chemical probes.

The recent advances of natural product chemistry certainly point to a bright future of this area in China. We feel confident that our talents will overcome the emerging challenges. The connection between structures and functions needs to be emphasized more than ever, which justifies natural product chemistry from the human heath perspective. Meanwhile, the creativity and productivity of natural product chemists are the key driving force to carry on the great momentum in the field; chemistry continues to stand in the premier position of this stage.

We owe a big debt of gratitude to all the contributors and reviewers for their time and efforts. We would like to thank Dr. Xuemei Zhang from the editorial office of Science China Chemistry for her kind help.

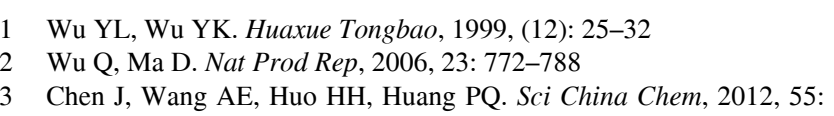

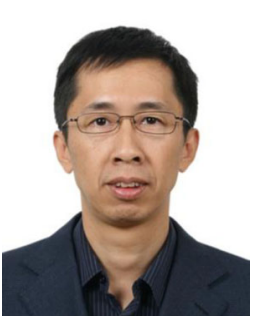

Prof. Qi-Yu Zheng received his B.Sc. degree in chemistry at Tongji University in 1994, M.S. and Ph.D. degrees in organic chemistry at the Institute of Chemistry, Chinese Academy of Sciences (ICCAS) in 1997 and 1999, respectively. Then he started his scientific career in ICCAS and has been promoted as a professor since 2010. From Apr. 2003 to Sep. 2005, he temporarily worked at Cornell University as a visiting scientist. His research interest includes synthesis and assembly of organic functional molecules. Now he is a program manager of the department of chemical sciences, the National Natural Science Foundation of China.

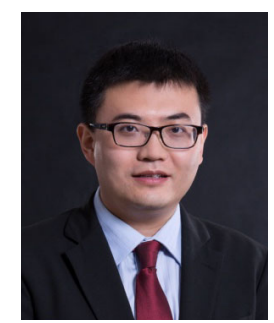

Prof. Ang Li received his B.Sc. from Peking University (China) in 2004, where he worked with Prof. Zhen Yang. He earned his Ph.D. at The Scripps Research Institute (US) in 2009, under the supervision of Prof. K. C. Nicolaou. After a brief stay as a research fellow at Institute of Chemical and Engineering Sciences (Singapore), he joined Shanghai Institute of Organic Chemistry, Chinese Academy of Sciences, as a professor in 2010. His current research focuses on the total synthesis of structurally and biologically interesting natural products. 